Concentrated Complete Fertilisers. One of the most interesting developments of the synthetic ammonia industry has been the manufacture of concentrated complete fertilisers containing nitrogen, phosphates and potash in suitable proportions and all soluble in water. One ton of such fertilisers supplies as much plant food as two tons of the ordinary mixed fertiliser of similar composition. They possess the obvious advantage of reducing freight and handling charges and cost of distribution to the land; they are granular in texture and very easy to sow, and they can be stored without risk of deterioration; further, the constituents are all soluble in water.

Another point claimed in their favour is that they contain little except the three fertilisers, nitrogen, phosphates and potash, while the ordinary fertilisers contain appreciable, and in some cases large, amounts of calcium, sulphur and other elements. It is possible that in some soils the absence of the additional substances might be a disadvantage, and a careful comparison of the new fertilisers with the old mixed fertilisers will be necessary to show that no disadvantage attends the use of the new compounds over a number of years.

It is obvious that if the concentrated fertilisers were used continuously over a number of years, increased attention would require to be given to liming.

\title{
Inter-Atomic Distances and Forces
}

$\mathrm{O}^{\mathrm{s}}$ September 11, in Section B (Chemistry) of the British Association meeting at Leicester, Dr. N. V. Sidgwick opened a discussion on "InterAtomic Distances and Forces in Molecules", with a review of the methods available for the determination of interatomic forces and distances.

$X$-Ray Measurements. Since the wave-length of $\mathrm{X}$-rays is of the same order as atomic distances, scattering of these rays gives information about the relative positions of atoms. This method of investigation has been applied extensively to solids, especially by Sir William and W. L. Bragg ever since its original suggestion by Laue, and has been extended more recently by Debye and others to liquids and vapours, where deformation of the molecules by crystal forces is absent. The method has been supplemented by use of electron waves.

Optical and Infra-red Spectra. Electronic energy changes correspond to spectral lines in the visible or ultra-violet region, but oscillation of molecules causes absorption of energy in quanta represented by lines in the infra-red. These differences may be imposed upon electronic changes to give the lines of a band spectrum. Rotational frequencies give lines in the far infra-red which, when imposed upon electronic and vibrational changes, give the fine structure lines of band spectra. From rotational quanta the moment of inertia of the molecules can be calculated; hence with known masses, inter-atomic distances and ultimately valency angles can be found. Oscillation frequencies indicate resistance to deformation of links.

Thermochemical Data. The energy given out when two atoms form a link is connected with the force constant of the link derived from its oscillation quanta; these are connected, however, with small changes only in the energy of the link, while the heat of formation represents the total energy associated with the link.

Dipole moments. These are produced whenever two unlike atoms form a link, and are due to unsymmetrical sharing of electrons. As resultant moments are obtained by compounding the simple vectors, observational values give indications of the valency angles. The values vary with the nature of the atoms, and with the nature and number of the links between them.

The properties of a link depend upon whether it is ionised or covalent, and, if covalent, upon whether it is single, double or treble. It is important to be able to distinguish the different kinds of links, and light has been thrown upon this by wave-mechanics. Measures of the lengths of covalent links accurate to about five per cent are now available and can be assigned in molecular structure. A double link is shorter than a single, and a triple link shorter than a double link between corresponding atoms. When, however, no link is present, atoms cannot approach so near to one another owing to electron repulsion. Atomic centres in the hydrogen molecule $\mathrm{H}_{2}$ are $0.75 \mathrm{~A}$. apart, but uncombined hydrogen atoms cannot normally approach within less than $2 \mathrm{~A}$. of each other. In methylene chloride there is room for the chlorine atoms if the $\mathrm{C}_{-}^{-} \mathrm{Cl}$ angle is the tetrahedral one, but the 'envelopes' interfere causing separation of the chlorine atoms. This is obviously important in questions of steric hindrance.

The theories which have been put forward to explain the covalent link were reviewed by Prof. J. E. Lennard-Jones. In 1927 Heitler and London applied new physical methods to the investigation of chemical linkages. The reactions of atoms to light indicate the energy states of the atoms. There is a natural tendency for a system to revert to its lowest energy state. Heitler and London considered that atoms were each in the lowest ground state when reacting; certain pairings of electron spins were thus possible, but the theory failed to explain either directed valencies or double bonds. Pauling and Slater suggested that it was necessary to consider other states of an atom when a second atom was near. Thus in carbon, two $2_{s}$ and two $2_{p}$ electrons were replaced by one $2_{s}$ and three $2_{p}$ electrons, enabling four shared pairs of electrons to be made up. The definite orbits of the Bohr atom are thus diffused by the merging of the neighbouring energy levels, and the superimposing of the four necessary orbits 
gave a symmetrical sphere indicative of the symmetry of the carbon atom. A displacement in electron intensity on one side of the nucleus led to the formation of three other corresponding positions of electron intensity symmetrically arranged, indicating the directed character of the valencies. Reactivity was assumed to be associated with intensity of electron charge.

'Resonance' or exchange energy was introduced to explain the energy of the covalent link; where resonance was possible between two atoms, the resulting system had a lower energy than either atomic state singly. Physical conception of this was difficult and it did not serve to explain the co-ordinate link. This can be explained, however, by the concept of molecular orbitals. All electrons in a structure are shared to some extent by all the nuclei. Those remaining close to their original nuclei are said to have atomic orbitals; those linking chemically form molecular orbitals, for example, in $\mathrm{Li}_{2}$ there are two pairs of electrons in atomic orbitals and two electrons in molecular orbitals. Regarding, say, the hydrogen atom as a 'hole' in space, the electron can fill it with a pattern as a sound vibration makes a pattern in a pipe. The bigger the 'hole' the more 'spread' the pattern and the lower the energy of the electron. When two atoms are concerned, chance of the leakage of electrons from one to the other is small when the atoms are far apart, but when the holes overlap, the electrons wander and take up the lowest possible energy pattern. Linking of atoms may be regarded as removing the nodes from the energy pattern and thus lowering the energy.

This theory explains better than that of Heitler and London (1) the nature of the covalent link, (2) partial sharing of electrons by unequal nuclei and (3) polyatomic molecules. Where the nuclei have a symmetrical arrangement, the electrons must take up a pattern conforming to the general symmetry of the molecule, and this will be the lowest energy pattern. Overtones are possible, however, giving nodes, and hence directional effects are developed. 'Lone pairs', for example, in $\mathrm{NH}_{3}$, may thus be represented by an excrescence in the electron pattern of a molecule which can supply deficiencies in other patterns, for example, $\mathrm{BH}_{3}$, giving a more symmetrical pattern than either and hence a lower energy state. Consideration of $\mathrm{C}, \mathrm{CH}, \mathrm{CH}_{2}$, etc. indicates an induced directed valency in an atom due to atoms already attached.

A means of determining interatomic distances is by the Fourier analysis of X-ray data. The method, which was described by Dr. J. M. Robertson, is laborious, but claims a higher accuracy than any other in use. The periodicity of scattering of electron density is represented by a Fourier series the coefficients of which are proportional to structure factors. By taking sufficient experimental determinations these coefficients can be calculated. Where complex molecules are concerned, this method is the most straightforward for the analysis of X-ray data. Applied to anthracene, by com- pounding three projections of the molecule, it gives regular plane hexagon rings. The nearest distance between molecules in this compound is $3 \cdot 7 \mathrm{~A}$. (it is $3.9 \mathrm{~A}$. in aliphatic hydrocarbons) and the distance across the cleavage plane is $4 \cdot 2 \mathrm{~A}$. The interatomic distance for carbon is $1.41 \mathrm{~A}$. Applied to symmetrical tetramethylbenzene, the method gives a $\mathrm{C}-\mathrm{C}$ distance from nucleus to side chain of $1.47 \mathrm{~A}$., which is the mean of the diamond (aliphatic) and graphite (aromatic) values, 1.54 A. and $1.41 \mathrm{~A}$. respectively. There is also evidence of repulsion of adjacent methyl groups.

Dr. J. D. Bernal dealt with the nature, accuracy and constancy of interatomic distances. The neutral atomic 'radius' represents the link distance where repulsion becomes appreciable and is in no sense a true radius. It does not occur in ionic compounds or metals although there is an average distance characteristic of the metallic state. In metalloids such as bismuth, link distances show covalency in one direction and metallic combination in another. Homopolar links are shorter than any others (from $\mathrm{H}_{2}, 0 \cdot 75 \mathrm{~A}$., to $\mathrm{I}_{2}, 2 \cdot 7 \mathrm{~A}$.). The shortest molecular distance is $2.76 \mathrm{~A}$. (in water). $\mathrm{Xe}-\mathrm{Xe}$ in the solid is $4.4 \mathrm{~A}$.

From the point of view of accuracy, Fourier analysis alone gives accurate values without chemical assumptions, but other methods are accurate enough for model building and can be used to eliminate improbable structures. They are not sufficiently accurate to calculate distortions. Intermolecular effects do not alter interatomic distances; intramolecular effects other than valency forces cause distortion, but bond angles rather than bond lengths are affected. The additive law for 'radii' is fairly true as a first approximation but needs correction when resonance is possible between two parts of the molecule. This may be due to the influence of an ionic binding upon a homopolar one. Distances are strictly additive when there is no dipole moment for the link, but marked difference in the electropolar character of the atoms, for example, $\mathrm{HF}$, causes shortening of the link. A truer kind of resonance is due to alternative forms of the molecule involving only changes in the kind of link between specified atoms, for example, variation in the double bond positions in the diphenyl molecule. The case of co-ordinated hydrogen presents special features. The energy of binding in $\left(\mathrm{OH}_{3}\right)^{\circ}$ formation is the same as in water : it is really an ionic binding with additional resonance energy.

The derivation of the force constant between the atoms of a diatomic oscillator from the characteristic frequencies indicated by the Raman effect, infra-red spectra or fine structure investigations was discussed by Mr. E. J. Bowen. This constant varies with the multiplicity of the link. For single links it is usually between 2. and 4, for double links it is about 9 and for triple links about 17 dynes $/ \mathrm{cm} . \times 10^{5}$. In triatomic molecules, there are three modes of vibration, and relative intensities of Raman and infra-red lines indicate which mode corresponds to a particular frequency. From the 
presence or absence of a $Q$ branch it is possible to determine whether the vibration is parallel to the axis of least inertia or not, but this requires high dispersion in infra-red measurements. Analogies from other systems with approximately equal energy quanta can also be used for associating modes of vibration with particular lines.

Mr. C. N. Hinshelwood described experiments on the decomposition of molecules in which the rate of decomposition varies with the pressure in a complex manner. Analysis of the results indicates that several modes of decomposition are possible, different modes predominating in different regions of pressure. The decomposition products are apparently the same in each case. This indicates alternative modes of vibration in the molecule and a possible line of attack on structural problems from kinetic experiments.

\section{Obituary}

\section{Prof. G. Embden}

$\mathrm{B}^{\mathrm{Y}}$ the death on July 25 last of Gustav Embden, professor of chemical physiology in the University of Frankfort-on-Main, science has suffered a very severe loss; one of the most inspiring teachers and workers has passed away at the height of his successful activity.

Gustav Embden was born in 1874 in Hamburg, of a distinguished family; he studied medicine in Freiburg and Strasbourg, where the personal influence of Hofmeister and of his own lifelong friend Bethe brought him (after a few years at Zurich with Gaule and Hoeber, and later in Frankfort with Paul Ehrlich) into the field of physiological and biochemical research. In Hofmeister's biochemical laboratory at Strasbourg, where he worked while he was a member of the staff of Ewald's physiological laboratory, he started the work on intermediary metabolism which occupied him throughout his life.

In 1904 Embden went to Frankfort as head of the chemical laboratory of the city hospital, and in 1909 this laboratory was developed, as were so many laboratories in German hospitals, into a well-equipped and excellent institution with much greater facilities for work than the average university laboratory at that time. As the head of this institute, Embden became in 1914 professor of "vegetative Physiologie" in the University, a position which was founded then: the name implies the physiology of chemical function in the animal body.

Embden's early papers dealt with a number of questions which were investigated by the liver perfusion method, greatly improved in his hands; with the formation of sugar in isolated liver, of sugar from amino acids, of aceto-acetic acid, and of acetone from fatty acids, and from amino acids, of amino acids out of nitrogen-free bodies, and with the formation and disappearance of lactic acid in the liver. He was probably the first to indicate that glucose can be formed from lactic acid and that these two substances are convertible into one another in either direction in the animal body : this idea has been developed into one of the most important in the chemical physiology of muscular activity.

From 1912 onwards the work of Embden and his school was concentrated on muscle chemistry. The discovery by Harden and Young of the rôle of phosphates in alcoholic fermentation led
Embden to the idea that, in the glycolysis of animal tissues also, phosphoric esters of carbohydrates must take part as intermediaries; this idea, put forward for the first time by Embden, finally proved, after a long and chequered history, to be correct. It was Embden himself, after twenty years, who brought this idea to a successful conclusion a few months before his death in a paper published early in 1933, in which he traced out the path of the intermediary processes in glycolysis; his scheme is most important for the further development of our ideas on glycolysis both in animals and in yeast cells.

The history of Embden's work on the intermediary rôle of phosphates in glycolysis is a dramatic one indeed: whole groups of laborious papers sometimes appeared as wholly erroneous because of experimental or theoretical mistakes or misinterpretation: sometimes, however, conclusions of his, already abandoned by himself or discredited by others, had to be revived. Embden's idea that a diphosphoric ester of hexose is an intermediary compound in lactic acid formation from glycogen in muscle tissue was finally proved to be correct, after this idea had for years been deserted by the author himself, and another ester, a hexose monophosphate, had been supposed by him to be the 'lactacidogen'. His idea that lactic acid formation is not the chemical change immediately connected with muscular contraction, and that this body is formed after contraction is over, also proved correct; and the strong opposition to this idea, which was held on very good experimental grounds by Hill and Meyerhof, had to be withdrawn when contraction without lactic acid formation was discovered by Lundsgaard, and when new experimental work by Embden and Lehnartz and by Meyerhof demonstrated beyond doubt the existence of delayed lactic acid formation after a muscle twitch.

The following important results of Embden's work on the chemistry of muscle must be quoted : the discovery of the occurrence in muscle of hexose monophosphoric ester (the Embden ester); the formation of Harden and Young's diphosphoric ester in muscle pulp in the presence of fluoride; the action of mineral ions on the changes in muscle pulp; the discovery of adenylic acid in this and other tissues. Embden first recognised that this latter compound is the precursor of the long-known inosinic acid in muscle, and this led him to his 